\title{
Maximal green sequences for arbitrary triangulations of marked surfaces (Extended Abstract)
}

\author{
Matthew R. Mills $\|^{\dagger}$ \\ Department of Mathematics, University of Nebraska - Lincoln, Lincoln, NE, USA
}

\begin{abstract}
In general, the existence of a maximal green sequence is not mutation invariant. In this paper we show that it is in fact mutation invariant for cluster quivers associated to most marked surfaces. We develop a procedure to find maximal green sequences for cluster quivers associated to an arbitrary triangulation of closed higher genus marked surfaces with at least two punctures. As a corollary, it follows that any triangulation of a marked surface with at least one boundary component has a maximal green sequence.

Résumé. En général, l'existence d'une suite verte maximale n'est pas invariante par mutation. Dans cet article, nous montrons qu'elle est invariante par mutation pour les carquois amassés associés à la plupart des surfaces marquées. Nous développons une méthode permettant de trouver des suites vertes maximales pour les carquois amassés associés à une triangulation arbitraire d'une surface marquée fermée de genre supérieur avec au moins deux ponctions. Nous obtenons comme corollaire que toute triangulation d'une surface marquée avec au moins une composante de bord admet une suite verte maximale.
\end{abstract}

Keywords. maximal green sequences, higher genus marked surfaces, cluster algebras

\section{Introduction}

Cluster algebras were introduced by Fomin and Zelevinsky in [14]. Cluster algebras have become an important tool in the study of many areas of mathematics and mathematical physics. They play a role in the study of Teichmüller theory, canonical bases, total positivity, Poisson Lie-groups, Calabi-Yau algebras, noncommutative Donaldson-Thomas invariants, scattering amplitudes, and representations of finite dimensional algebras.

One very important property of a quiver associated to a cluster algebra is whether or not it has a maximal green sequence. Quiver mutation is a transformation of a quiver, determined by a choice of a vertex of the quiver, into a new quiver. A maximal green sequence is a certain sequence of quiver mutations given by a sequence of vertices of the quiver. The idea of maximal green sequences of cluster mutations was introduced by Keller in [18]. He explored quantum dilogarithm identities by utilizing these sequences in the

\footnotetext{
${ }^{\dagger}$ Email: matthew. mills@huskers. unl . edu. Supported by University of Nebraska - Lincoln, and by NSA grant H9823014-1-0323.

1365-8050 @ 2016 Discrete Mathematics and Theoretical Computer Science (DMTCS), Nancy, France
} 
explicit computation of noncommutative Donaldson-Thomas invariants of quivers which were introduced by Kontsevich and Soibelman in [19]. If a quiver with potential has a maximal green sequence, then its associated Jacobi algebra is finite dimensional [3, 17]. In [2] an explicit construction of a cluster category from a quiver with potential whose Jacobian algebra is finite dimensional is given.

The existence of a maximal green sequence for a quiver also seems to be related to whether the cluster algebra $\mathcal{A}$ it generates is equal to its upper cluster algebra $\mathcal{U}$. Gross, Hacking, Keel and Kontsevich showed that if $\mathcal{A}=\mathcal{U}$ and a maximal green sequence exists, then the Fock- Goncharov canonical basis conjecture holds [16]. It is still unknown as to whether or not $\mathcal{A}=\mathcal{U}$ for closed higher genus surfaces with at least two punctures and punctured closed spheres. For all other quivers from marked surfaces it is known that $\mathcal{A}=\mathcal{U}$ if and only if there exists a quiver with a maximal green sequence. See [7] and references therein for more information on $\mathcal{A}=\mathcal{U}$.

The main result in this paper focuses on the existence of maximal green sequences for quivers that are associated to triangulations of surfaces.

Theorem 1.1 Suppose that a marked surface $\Sigma$ is:

(A) of genus at least one with at least two punctures;

(B) or of arbitrary genus with at least one boundary component.

Then for any triangulation of $\Sigma$ there exists a maximal green sequence for the associated quiver.

The proof of Theorem 1.1 $(A)$ is an explicit construction of maximal green sequences for these surfaces. We only give a brief overview of the construction in this paper. A more detailed proof will be given in a later work. The proof for $(B)$ follows from $(A)$ together with a theorem of Muller that we recall here as Theorem 4.4 We give a sketch of this proof in Section 4.2

It was shown by Ladkani that quivers associated to once-punctured closed surfaces of genus at least one do not admit maximal green sequences [20]. We do not expect that it will be difficult to expand Theorem 1.1 to include the case of the punctured closed sphere and it will be done in the near future.

It is straightforward to see from the definition of quiver mutation (formally stated in Definition 2.2 ) that mutation imposes an equivalence relation on the set of all quivers. For a quiver $Q$ we let $M u t(Q)$ denote the equivalence class of $Q$ under this relation. A quiver $Q$ is said to be of finite mutation type if $M u t(Q)$ is finite.

Conjecture 1.2 Let $Q$ be a quiver of finite mutation type, then a maximal green sequence exists for every quiver in $M u t(Q)$, or there is no maximal green sequence for any quiver in $M u t(Q)$.

Muller showed that in general the existence of a maximal green sequence is not mutation invariant [21]. It is already known that every quiver of finite type has a maximal green sequence [3]. The existence of maximal green sequences for special triangulation of various marked surfaces has been shown in many papers [1, 5, 6]. This work shows that Conjecture 1.2 holds, for all cluster quivers arising from surfaces except for those of closed spheres. It is known that all finite mutation type quivers arise from triangulations of surfaces except for the rank 2 case and 11 exceptional cases. Among these exceptional cases it has been shown that there exists a quiver with a maximal green sequence for all but $\mathbb{X}_{7}[1]$. It was shown by Seven in [22] that $\mathbb{X}_{7}$ has no maximal green sequence. In [15] Garver and Musiker give a combinatorial approach to construct maximal green sequences for type $\mathbb{A}$ quivers, which are exactly the quivers associated to triangulations of unpunctured disks. Cormier et al., give an explicit construction of 
minimal length maximal green sequences for this case in [8]. In [4] Brüstle, Hermes, Igusa, and Todorov use semi-invariants to prove two conjectures about maximal green sequences.

In Section 2 we give background on quivers and maximal green sequences. In Section 3 we give background on marked surfaces and their triangulations. In Section 4 we give an outline of the proofs of Theorem 1.1 We then give examples in Section 5

\section{Quivers and maximal green sequences}

We recall the definitions from [17], but use the setup given in [3].

Definition 2.1 A (cluster) quiver is a directed graph with no loops or 2-cycles. An ice quiver is a pair $(Q, F)$ where $Q$ is a quiver and $F$ is a subset of the vertices of $Q$ called frozen vertices; such that there are no edges between frozen vertices. If a vertex of $Q$ is not frozen it is called mutable. For convenience, we assume that the mutable vertices are labelled $\{1, \ldots, n\}$, and frozen vertices are labeld by $\{n+1, \ldots, n+m\}$.

Definition 2.2 Let $(Q, F)$ be an ice quiver, and $k$ a mutable vertex of $Q$. The mutation of $(Q, F)$ at vertex $k$ is denoted by $\mu_{k}$, and is a transformation $(Q, F)$ to a new ice quiver $\left(\mu_{k}(Q), F\right)$ that has the same vertices, but making the following adjustment to the edges:

1. For every 2-path $i \rightarrow k \rightarrow j$, add a new arrow $i \rightarrow j$.

2. Reverse the direction of all arrows incident to $k$.

3. Delete any 2-cycles created during the first two steps, and any arrows between frozen vertices.

Mutation at a vertex is an involution, and an equivalence relation. We define $\operatorname{Mut}(Q)$ to be the equivalence class of all quivers that can be obtained from $Q$ by a sequence of mutations.

Definition 2.3 Let $Q_{0}$ be the set of vertices of $Q$. The framed quiver associated with a quiver $Q$ is the ice quiver $\left(\hat{Q}, Q_{0}^{\prime}\right)$ such that:

$$
\begin{gathered}
Q_{0}^{\prime}=\left\{i^{\prime} \mid i \in Q_{0}\right\}, \quad \hat{Q}_{0}=Q_{0} \sqcup Q_{0}^{\prime} \\
\hat{Q}_{1}=Q_{1} \sqcup\left\{i \rightarrow i^{\prime} \mid i \in Q_{0}\right\}
\end{gathered}
$$

Since the frozen vertices of the framed quiver are so natural we will simplify the notation and just write $\hat{Q}$. Now we must discuss what is meant by red and green vertices.

Definition 2.4 Let $R \in M u t(\hat{Q})$.

A mutable vertex $i \in R_{0}$ is called green if

$$
\left\{j^{\prime} \in Q_{0}^{\prime} \mid \exists j^{\prime} \rightarrow i \in R_{1}\right\}=\emptyset .
$$

It is called red if

$$
\left\{j^{\prime} \in Q_{0}^{\prime} \mid \exists j^{\prime} \leftarrow i \in R_{1}\right\}=\emptyset .
$$

The result that every mutable vertex in $R_{0}$ is either red or green is due to [9] and then in a more general setting in [16].

Definition 2.5 A green sequence for $Q$ is a sequence $\boldsymbol{i}=\left(i_{1}, \ldots, i_{l}\right) \subset Q_{0}$ such that $i_{1}$ is green in $\hat{Q}$ and for any $2 \leq k \leq l$, the vertex $i_{k}$ is green in $\mu_{i_{k-1}} \circ \cdots \circ \mu_{i_{1}}(\hat{Q})$. A green sequence $\boldsymbol{i}$ is called maximal if every mutable vertex in $\mu_{i_{l}} \circ \cdots \circ \mu_{i_{1}}(\hat{Q})$ is red. 

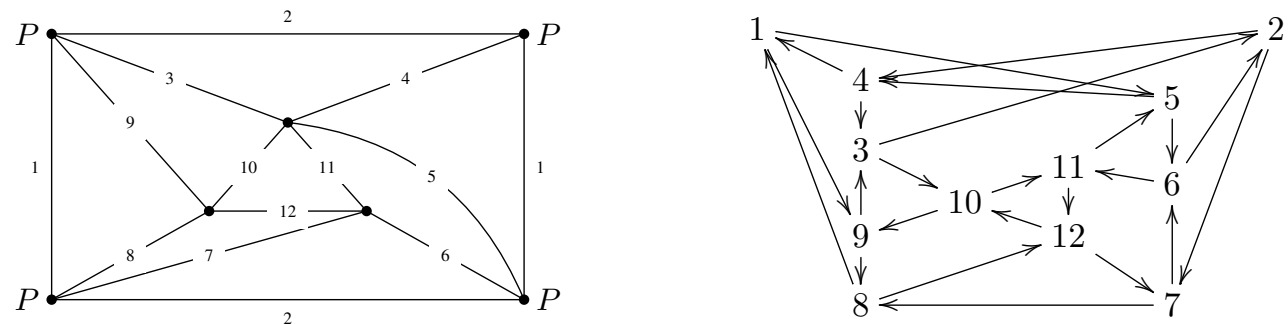

Fig. 1: A triangulation $T_{1}$ of $\Sigma_{1}=\{1,0,4, \emptyset\}$ (left), and the corresponding quiver $Q_{T_{1}}$ (right).

\section{Marked surfaces and their triangulations}

To begin the section we recall the definition of a marked surface given in [12]. Let $S$ be an orientable 2-dimensional Riemann surface with or without boundary. We designate a finite number of points $M$ in the closure of $S$ as marked points. We require at least one marked point on each boundary component. We call marked points in the interior of $S$ punctures. Together the pair $\Sigma=(S, M)$ is called a marked surface. For technical reasons we exclude the cases when $\Sigma$ is one of the following:

- a sphere with less than four punctures;

- an unpunctured or once punctured monogon;

- an unpunctured digon; or

- an unpunctured triangle.

Note that the construction allows for spheres with four or more punctures, but we do not discuss this case in this note.

Up to homeomorphism a marked surface is determined by four things. The first is the genus $g$ of the surface. The second is the number of boundary components $b$ of $S$. The third is the number of punctures $p$ in $M$, and the fourth is the set $m=\left\{m_{i}\right\}_{i=1}^{b}$ where $m_{i} \in \mathbb{Z}_{>0}$ denotes the number of marked points on the $i$ th boundary component of $S$. We will commonly say a surface $\Sigma=\{g, b, p, m\}$ when we mean that $\Sigma$ is a surface in this homeomorphism class.

Definition 3.1 An arc $\gamma$ in $(S, M)$ is a curve in $S$ such that:

- The endpoints of $\gamma$ are in $M$.

- $\gamma$ does not intersect itself, except that its endpoints may coincide.

- $\gamma$ is disjoint from $M$ and the boundary of $S$, except at its endpoints.

- $\gamma$ is not isotopic to the boundary, or the identity.

An arc is called a loop if its two endpoints coincide. Each arc is considered up to isotopy. Two arcs are called compatible if there exists two arcs in their respective isotopy classes that do not intersect in the interior of $S$. 

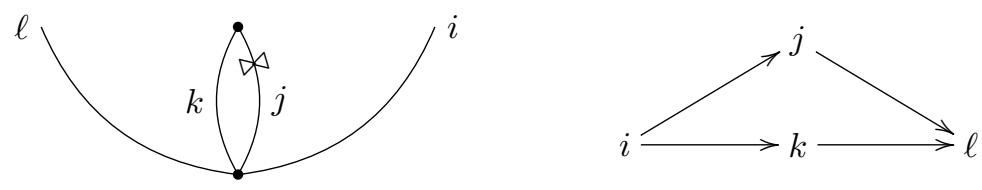

Fig. 2: A special situation in the construction of a quiver from a triangulation.

Definition 3.2 A taggd arc is constructed by taking an arc that does not cut out a once-punctured monogon and marking or "tagging" its ends as either plain or notched so that:

- an endpoint lying on the boundary of $S$ is tagged plain; and

- both ends of a loop must be tagged in the same way.

We use a $\bowtie$ to denote the tagging of an arc in figures. Two tagged arcs are considered compatible if:

- Their underlying untagged arcs are the same, and their tagging agrees on exactly one endpoint.

- Their underlying untagged arcs are distinct and compatible, and any shared endpoints have the same tagging.

A maximal collection of pairwise compatible tagged arcs is called a (tagged) triangulation of $(S, M)$.

Each triangulation $T$ of $(S, M)$ gives rise to a quiver, $Q_{T}$, by the following construction. For each arc $\alpha$ in a triangulation $T$ add a vertex $v_{\alpha}$ to $Q_{T}$. If $\alpha_{i}$ and $\alpha_{j}$ are two edges of a triangle in $T$ with $\alpha_{j}$ following $\alpha_{i}$ in a clockwise order, then add an edge to $Q_{T}$ from $v_{\alpha_{i}} \rightarrow v_{\alpha_{j}}$. If $\alpha_{k}$ and $\alpha_{j}$ have the same underlying untagged arc we refer you to Figure 2 for the construction in this situation. Note that the quiver is the same whether $\alpha_{j}$ or $\alpha_{k}$ is tagged, and distinct triangulations may yield the same quiver.

We now define the analog of quiver mutation for triangulations of a marked surface.

Definition 3.3 A flip is a transformation of a triangulation that removes an arc $\gamma$ and replaces it with a (unique) different arc $\gamma^{\prime}$ that, together with the remaining arcs, forms a new triangulation $T^{\prime}$. In this case we define $\mu_{\gamma}(T)=T^{\prime}$. This makes sense by the following lemma.

Lemma 3.4 [12 Lemma 9.7] Let $T$ and $T^{\prime}$ be two triangulations related by a flip of an arc $\gamma$. Suppose $\gamma$ corresponds to vertex $k$ of $Q_{T}$, then $Q_{T^{\prime}}=\mu_{k}\left(Q_{T}\right)$.

Remark 3.5 Thurston's theory of laminations and shear coordinates provide a way to keep track of frozen variables on a surface, but for brevity we do not discuss it here. The interested reader can see [1] 13] for more information.

It is helpful for the discussion in Section 4 to refer to an arc as being either green or red. Suppose that $T$ is a triangulation of some marked surface and $Q_{T}$ is its corresponding quiver. Let $\mu$ be some sequence of mutations. We say that an $\operatorname{arc}$ in $\mu(T)$ is green (resp. red) if its corresponding vertex in $\mu\left(Q_{T}\right)$ is green (resp. red). 


\section{Existence of maximal green sequences.}

\subsection{Construction of sequences for higher genus closed surfaces}

In this section we let $\Sigma=\{g, 0, p, \emptyset\}$, where $g \geq 1$ and $p \geq 2$. It was shown by Ladkani in [20] that any once-punctured higher genus surface has no maximal green sequence.

In any triangulation when there are at least three arcs (none of which are loops) incident to a puncture, these arcs form an oriented cycle in the corresponding quiver. The mutation sequences given in this paper rely heavily on the following lemma which gives a maximal green sequence for when each vertex in the oriented cycle is green.

Lemma 4.1 [5. Lemma 4.2] Let $C$ be a quiver that is an oriented $n$-cycle with vertices labeled $c_{i} i=$ $1, \ldots, n$, with $c_{i} \rightarrow c_{i-1}$ for $2 \leq i \leq n$ and $c_{1} \rightarrow c_{n}$. Define a sequence

$$
\chi\left(c_{n}, c_{n-1}, \ldots, c_{2}, c_{1}\right)=c_{n} c_{n-1} \cdots c_{2} c_{1} c_{3} c_{4} \cdots c_{n-1} c_{n} .
$$

Then $\chi$ is a maximal green sequence for $C$. Furthermore, after applying $\chi$ to $C$ the resultant quiver is still an oriented cycle with $c_{2}$ and $c_{1}$ interchanged.

When we say that vertices $i$ and $j$ are interchanged we mean that there exists a relabelling of the quiver that fixes the label on vertex $k$ if $k \neq i, j$; but relabels vertex $i$ with $j$ and vice versa. In the degenerate case when there are exactly two arcs incident to a puncture, the corresponding vertices are not connected by an edge. However we will still refer to these two arcs as a cycle and the mutation seqence $\mu=\left(c_{1}, c_{2}\right)$ is a maximal green sequence that interchanges the arcs corresponding to $c_{1}$ and $c_{2}$.

There is a subset of the triangulations of higher genus surfaces where fundamental loops are arcs in the triangulation. We give a name to triangulations that have the nicest version of this property.

Definition 4.2 Let $T$ be a triangulation of a surface $\Sigma$ with genus $g \geq 1$. A fundamental loop of $\Sigma$ is a loop without self-crossings that is not homotopy equivalent to a point on the unmarked surface. We say that $T$ is a delta-triangulation if there exists a puncture $P$ such that there exists $2 g$ fundamental loops $\psi_{1}, \phi_{1}, \ldots, \psi_{g}, \phi_{g}$ in $T$ based at $P$ which form a standard fundamental $4 g$-polygon

$$
\phi_{1}, \psi_{1} \phi_{1}^{-1}, \psi_{1}^{-1}, \ldots, \phi_{g}, \psi_{g} \phi_{g}^{-1}, \psi_{g}^{-1}
$$

in the unversal cover of $\Sigma$. We use $\partial^{P} T$, or just $\partial T$ when the puncture is irrelevant, to denote the set of all fundamental loops in $T$ based at puncture P. In other words we have;

$$
\partial^{P} T=\{\alpha \in T \mid \alpha \text { is a fundamental loop of } \Sigma \text { based at puncture P. }\}
$$

and $T$ is a delta-triangulation if and only if there exists a puncture $P \in \Sigma$ such that a subset of arcs in $\partial^{P} T$ form a standard fundamental polygon.

In Figure 1 we have $\partial^{P} T_{1}=\{1,2\}$ and $T_{1}$ is a delta-triangulation. In Figure 8 we have $\partial^{P} T_{2}=\{1,2\}$ and $T_{2}$ is not a delta-triangulation. In Figure 9 we have $\partial^{P} T_{3}=\{1,2,3,4\}$ and $T_{3}$ is delta-triangulation. In Figure 10 we have $\partial^{P} T_{4}=\{1,2\}$ and $T_{4}$ is a delta-triangulation.

We give a brief overview of a maximal green sequence for the quivers associated to triangulations of $\Sigma$. When we refer to mutation of an arc we mean to mutate the vertex of the quiver corresponding to the arc and to flip the arc in the triangulation. We omit the proof that the mutation sequence described in the following steps is a maximal green sequence for brevity. 
1. Mutating to a delta-triangulation. Choose a puncture $P$ of $\Sigma$. If our initial triangulation is a deltatriangulation and there is exactly one puncture $R$ that is not encosed in a monogon we cannot choose $P$ to be $R$. If our initial triangulation of $\Sigma$ was not a delta-triangulation, then we first mutate to a deltatriangulation. Suppose that we want to obtain a fundamental loop $\gamma$ based at a puncture $P$. If we superimpose $\gamma$ onto $T$ and label the distinct arcs crossed by traversing $\gamma$ as $\alpha_{1}, \cdots, \alpha_{n}$. Then the sequence $\left(\alpha_{1}, \alpha_{n} \alpha_{2}, \alpha_{n-1}, \ldots, \alpha_{\left\lceil\frac{n+1}{2}\right\rceil}\right)$ gives a green sequence to obtain that fundamental loop in your triangulation. We apply this until we obtain a delta-triangulation. We call this mutation sequence $\mu_{P}$. Note that if $T$ is a delta-triangulation based at $P$ then $\mu_{P}$ is empty. One remarkable property of this algorithm is that if $Q$ is some other puncture in $\Sigma$, then even if $T$ is a delta-triangulation with loops based at $P$, we can begin a maximal green sequence by first mutating to $\mu_{Q}(T)$.

2. Separating of "interior" punctures. We call two punctures $Q$ and $R$ of $\Sigma$ separated in a triangulation $T$ if there is no arc with one endpoints at $Q$ and the other at $R$ in $T$. Let $\mathcal{P}$ denote the set of all punctures of $\Sigma$ except for $P$ and any punctures enclosed by monogons. In this step of the maximal green sequence we want to separate each pair of punctures in $\mathcal{P}$. Call this mutation sequence $\mu_{\text {sep }}$.

3. Transfering the basepoint of monogons. Punctured monogons require special attention in the construction of our maximal green sequence. By separating the punctures of $\mathcal{P}$ we have that any punctured monogon will be cut out by a digon between $P$ and some puncture $Q \in \mathcal{P}$. At this point we mutate the monogons based at $Q$ and will handle monogons based at $P$ in Step 6. We may switch the basepoint of these monogons from $Q$ to $P$ by mutating the arcs that are incident to $Q$ in a particular order and leaving any arcs between the punctures inside the monogon unaffected. We do this for all punctured monogons in the triangulation based at some $Q \in \mathcal{P}$ and call this mutation sequence $\mu_{M}$.

The point of this step is so that we can get an oriented cycle around the punctures of $\mathcal{P}$. When there is a monogon based at a puncture the corresponding cycle in the quiver passes through the vertex corresponding to the monogon twice and Lemma 4.1 does not apply.

4. Mutating cycles around punctures. After separating the punctures of $\mathcal{P}$ and moving the monogons basepoints to $P$, we have two possible cases for a puncture $S \in \mathcal{P}$; (1) every arc incident to $S$ is green and has its other endpoint at $P$; (2) $S$ is incident to exactly one green and one red arc where the other endpoint of each arc is at $P$ and differ only by the red arc being tagged at $S$. In the first case this means that in the corresponding quiver there is an oriented cycle of green vertices. We apply Lemma 4.1 to each of these cycles in $\mu_{M} \mu_{\text {sep }} \mu_{P}(T)$. The second case arises if $S$ initially had exactly one arc $\alpha$ with endpoints at $S$ and $P$, then during the separation step we would have introduced an $\operatorname{arc} \beta$ that only differs from $\alpha$ by having the opposite tagging at $Q$. In this case we only mutate $\alpha$ since $\beta$ is already tagged at $Q$. Call this mutation sequence $\mu_{\text {cycle }}$.

5. Mutating back to our initial triangulation. Note that some arcs may have been interchanged, by the application of $\mu_{\text {cycle }}$. We assume that this is not the case for any arc that was mutated during $\mu_{\text {sep }}$ or $\mu_{P}$. This is possible by starting the mutation of the cycle at an appropriate vertex. Mutation is an involution, so we have that the inverse of a mutation sequence is just the reverse of the mutation sequence. At this point in our mutation sequence $\left(\mu_{M} \mu_{\text {sep }} \mu_{P}\right)^{-1}=\mu_{P}^{-1} \mu_{\text {sep }}^{-1} \mu_{M}^{-1}$ is a green mutation sequence for $\mu_{\text {cycle }} \mu_{M} \mu_{\text {sep }} \mu_{P}(T)$. We apply this sequence now with slight a change. If we are in the situation (2) described in the previous section then we omit the arc that is already tagged at the puncture from $\mu_{\text {sep }}^{-1}$.

It is worth while to note that in this stage of the green sequence all of the punctures of $\mathcal{P}$ have changed their taggings. Any arcs whose endpoints exclusively belong to $\mathcal{P}$ will no longer have to be mutated. 

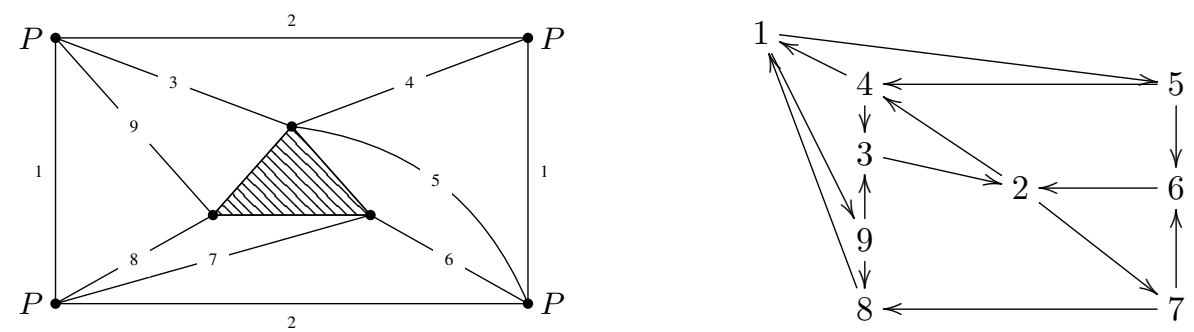

Fig. 3: A triangulation $T^{*}$ of $\Sigma^{*}=\{1,1,1,\{3\}\}$ (left), and the corresponding quiver (right) that we extend to the triangulation in Figure 1 . Note that this quiver is an induced subquiver of the one in Figure 1

Furthermore, since any monogon based at puncture of $\mathcal{P}$ will no longer need to be mutated the quiver is effectively broken into disjoint subquivers. We now turn our attention to flipping the tagging at puncture $P$, and conclude with the punctures inside of a monogon.

6. Preparing puncture $P$. First, we apply a mutation sequence annalogous to the one given above to any monogon based at $P$ to transfer the monogon to the opposite puncture in the digon enclosing it. Call this sequence $\mu_{M_{P}}$. We then mutate any other arcs that are loops based at $P$. First we mutate the loops that are not arcs in $\partial^{P}(T)$ in a sequence called $\mu_{H}$. These arcs will no longer be loops at $P$. We then mutate the $\operatorname{arcs}$ of $\partial^{P}(T)$ in a sequence called $\mu_{B}$ so they are no longer loops at $P$. In general, it is necesarry to do this in two different steps.

7. Cycle around $\mathbf{P}$. In the current state of the quiver/triangulation the only green arcs are incident to puncture $P$ and form an oriented cycle in the quiver. We apply Lemma 4.1 to this cycle and call it $\mu_{C}$. We now apply $\mu_{B}^{-1}$ followed by $\mu_{H}^{-1}$ and finally $\mu_{M_{P}}^{-1}$.

8. Punctures enclosed in a monogon. We now apply a mutation sequence to the arcs enclosed in the punctured monogon. We can apply a sequence similar to that of Steps 2-5 to make the arcs in the interior of the monogon red. We call this mutation sequence $\mu_{I}$.

Putting together the previous steps we have that the sequence

$$
\mu_{I} \mu_{M_{P}}^{-1} \mu_{H}^{-1} \mu_{B}^{-1} \mu_{C} \mu_{B} \mu_{H} \mu_{M_{P}} \mu_{P}^{-1} \mu_{\text {sep }}^{-1} \mu_{M}^{-1} \mu_{\text {cycle }} \mu_{M} \mu_{\text {sep }} \mu_{P},
$$

is a maximal green sequnce for $Q_{T}$. Note that with function notation the sequence is read from right to left. Also note that some of mutation sequences may be empty.

\subsection{Existence for surfaces with nonempty boundary}

We first recall the definition of an induced subquiver and a theorem from Muller.

Definition 4.3 Given a subset $V$ of vertices of a quiver $Q$, the induced subquiver, is the quiver with vertex set $V$ and edges consisting of the edges between pairs of vertices in $V$ that are in $Q$.

Theorem 4.4 [21] Lemma 1.4.1] If a quiver admits a maximal green sequence, then any induced subquiver admits a maximal green sequence.

By the previous theorem it suffices to show that the quiver obtained from any triangulation of a surface with boundary is an induced subquiver of a closed surface with at least two punctures. 


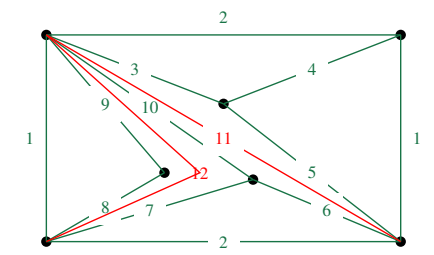

Fig. 4: $\mu_{\text {sep }}\left(T_{1}\right)$

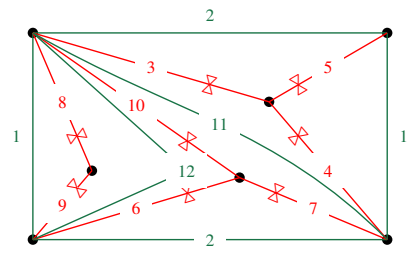

Fig. 5: $\mu_{\text {cycle }} \mu_{\text {sep }}\left(T_{1}\right)$

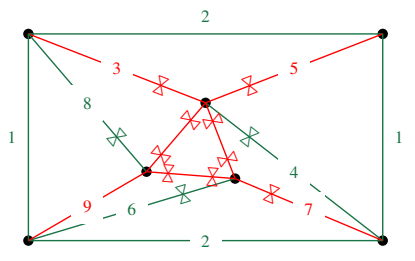

Fig. 6: $\mu_{\text {sep }}^{-1} \mu_{\text {cycle }} \mu_{\text {sep }}\left(T_{1}\right)$

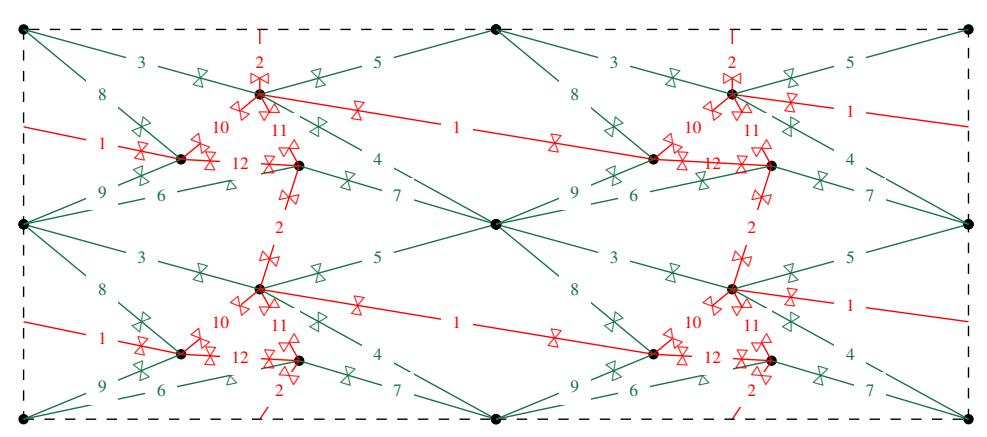

Fig. 7: $\mu_{B} \mu_{\text {sep }}^{-1} \mu_{\text {cycle }} \mu_{\text {sep }}\left(T_{1}\right)$

Suppose $\Sigma=(S, M)=\{g, b, p, m\}$ is a surface with boundary and $g \geq 1$. Let $\bar{\Sigma}=\{g, 0, p+$ $N(b, m), \emptyset\}$, where $N(b, m)$ is an integer determined by the number of boundary components of $\Sigma$ and the number of marked points on them. We show that for any triangulation $T$ of $\Sigma$, we can embed $T$ into $\bar{\Sigma}$ and extend it to a triangulation $\bar{T}$ of $\bar{\Sigma}$ by replacing a boundary component $b_{i}$ with a (possibly punctured) $m_{i}$-gon. By our previous work we know that $Q_{\bar{T}}$ has a maximal green sequence so we conclude the proof for the boundary case by showing that $Q_{T}$ is an induced subquiver of $Q_{\bar{T}}$. We also adress the case of punctured disks; possibly with more than one boundary component. If $\Sigma=\{0, b, p, m\}$ with $b \geq 1$, it is easy to see that any triangulation $T$ of $\Sigma$ can be embedded into a torus and extend to a triangulation so it again follows that $Q_{T}$ has a maximal green sequence.

\section{Examples}

In this section we give examples following the outline given in the previous section. We refer the reader to the java applet by Keller if they wish to verify the examples. The applet can be found at http: //webusers.imj-prg.fr/ bernhard.keller/quivermutation/

Example 1. In Figure 1 we give a delta-triangulation $T_{1}$ of $\Sigma=\{1,0,4, \emptyset\}$. A maximal green sequence for $Q_{T_{1}}$ is given by

$$
i_{1}=(10,11,12,10,7,6,10,9,8,3,5,4,3,12,11,10,1,2,4,7,5,8,3,6,9,3,8,5,7,4,2,1) .
$$

We draw the corresponding triangulations at different stages of $i_{1}$ in Figures 4 -7 7 We do not show the application of $\mu_{C}$ or $\mu_{B}^{-1}$. We also provide a decomposition of $i_{1}$ into the nonempty mutation sequences 

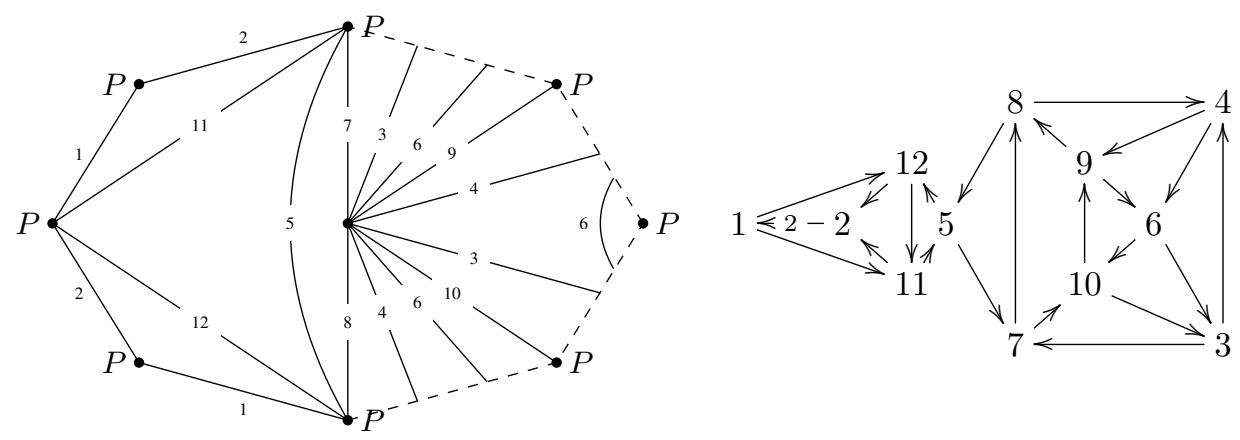

Fig. 8: A triangulation $T_{2}$ of $\Sigma_{2}=\{2,0,2, \emptyset\}$ (left), and the corresponding quiver $Q_{T_{2}}$ (right).
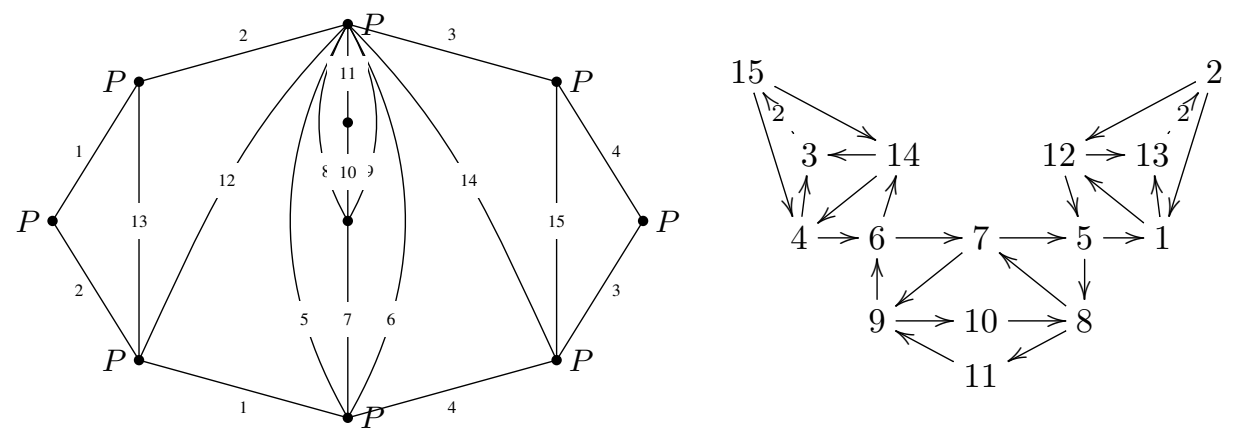

Fig. 9: A triangulation $T_{3}$ of $\Sigma=\{2,0,3, \emptyset\}$ (left), and the corresponding quiver $Q_{T_{3}}$ (right).

given in Section 4 . It is as follows; $\mu_{\text {sep }}=(10,11,12), \mu_{\text {cycle }}=(10,7,6,10,9,8,3,5,4,3,11,12,10)$, $\mu_{C}=(4,7,5,8,3,6,9,3,8,5,7,4)$, and finally $\mu_{B}=(1,2)$. Note that $\mu_{\text {cycle }}$ is composed of the three green cycles around the punctures; one of which is $(9,8)$.

In Figure 3 we give a triangulation $T^{*}$ of $\Sigma^{*}=\{1,1,1,\{3\}\}$ which extends to $T$. Note that the quiver associated to $T^{*}$ is an induced subquiver of $Q_{T_{1}}$ and is obtained by taking all the vertices of $Q_{T_{1}}$ except for 10,11, and 12. Therefore by Theorem 4.4 we have that $Q_{T^{*}}$ has a maximal green sequence.

Example 2 In Figure 8 we give a triangulation $T_{2}$ of a surface $\Sigma_{2}=\{2,0,2, \emptyset\}$.

$i_{2}=(6,3,4,10,6,9,7,8,9,6,10,4,3,6,5,11,12,1,2,7,11,5,12,8,10,9,8,12,5,11,7,2,1,12,11,5)$.

Again, the decomposition of the sequence is provided. $\mu_{P}=(6,3,4), \mu_{\text {cycle }}=(10,6,9,7,8,9,6,10)$, $\mu_{H}=(5,11,12), \mu_{B}=(1,2)$, and $\mu_{C}=(7,11,5,12,8,10,9,8,12,5,11,7)$.

Example 3 In Figure 9 we give a triangulation $T_{3}$ of the surface $\Sigma_{3}=\{2,0,3, \emptyset\}$ that was studied in [6]. The maximal green sequence given in this paper is shorter then the one appearing there. The sequence given here has length 44, whereas the maximal green sequence for this quiver given in [6] has length 52. The maximal green sequence is given by

$$
i_{3}=(10,8,7,9,8,11,5,12,13,6,14,15,1,2,3,4,
$$



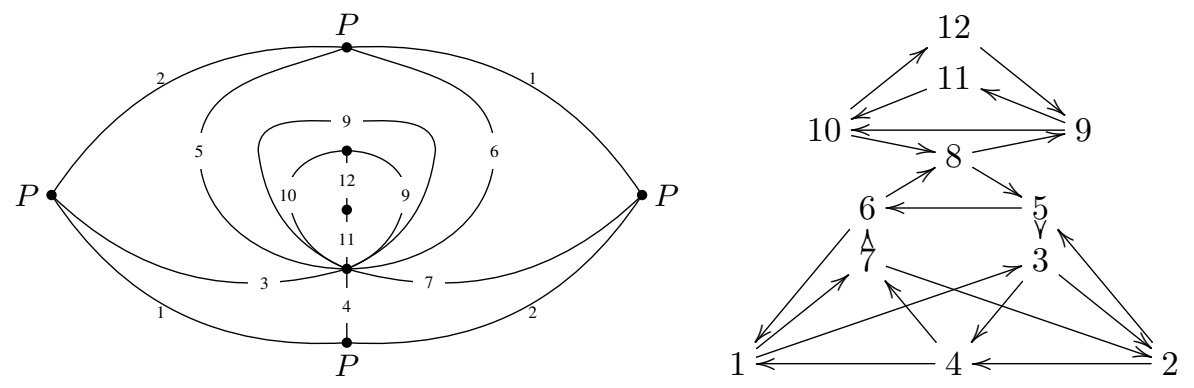

Fig. 10: A triangulation $T_{4}$ of $\Sigma_{4}=\{1,0,3, \emptyset\}$ (left), and the corresponding quiver $Q_{T_{4}}$ (right).

$$
5,8,10,7,6,15,14,9,12,13,9,14,15,6,7,10,8,5,4,3,2,1,15,14,6,12,13,5) .
$$

The sequence is decomposed into the steps from Section 4 as follows;

$\mu_{\text {sep }}=(10), \mu_{\text {cycle }}=(8,7,9,8,11), \mu_{H}=(5,12,13,6,14,15), \mu_{B}=(1,2,3,4)$, and

$\mu_{C}=(5,8,10,7,6,15,14,9,12,13,9,14,15,6,7,10,8,5)$. Note that in this mutation sequence $\mu_{\text {sep }}^{-1}$ is empty.

Example 4. In Figure 10 we give a triangulation of $\Sigma_{4}=\{1,0,3, \emptyset\}$ with a punctured monogon.

$$
i_{4}=(8,9,11,10,6,5,3,4,7,3,5,6,10,11,9,8,1,2,5,6,3,7,4,3,6,5,2,1,12,9,10,11) .
$$

The decomposition of $i_{4}$ is as follows; $\mu_{M}=(8,9,11,10), \mu_{\text {cycle }}=(6,5,3,4,7,3,5,6), \mu_{B}=(1,2)$, $\mu_{C}=(5,6,3,7,4,3,6,5)$, and $\mu_{I}=(12,9,10,11)$.

\section{Acknowledgements}

The author would like to thank Kyungyong Lee for many helpful discussions, Pierre-Guy Plamondon for translating the abstract to French, and Khrystyna Serhiyenko for helpful suggestions to improve the manuscript.

\section{References}

[1] Alim, M., Cecotti, S., Cordova, C., Espahbodi, S., Rastogi, A., Vafa, C., BPS Quivers and Spectra of Complete $N=2$ Quantum Field Theories, Communications in Mathematical Physics. 323, (2013) 1185-1127.

[2] Amiot, C., Cluster categories for algebras of global dimension 2 and quivers with potential, Annales de l'institut Fourier 59 (2009), no. 6, 2525-2590.

[3] Brüstle, T., Dupont, G., Pérotin, M., On Maximal green Sequences, International Mathematics Research Notices. 16 (2014), 4547-4586.

[4] Brüstle, T., Hermes, S., Igusa, I., Todorov, G., Semi-invariant pictures and two conjectures on maximal green sequences. arXiv:1503.07945. 
[5] Bucher, E., Maximal green Sequences for cluster algebras associated to the $n$-torus arXiv:1412.3713.

[6] Bucher, E., Mills, M., Maximal Green Sequences for Cluster Algebras Associated to the Orientable Surfaces of Genus $n$ with Arbitrary Punctures, arXiv:1503.06207.

[7] Canakci, I., Lee, K., Schiffler, R., On cluster algebras from unpunctured surfaces with one marked point, Proc. Amer. Math. Soc. Ser. B 2 (2015), 35-49.

[8] Cormier, E., Dillery, P., Resh, J., Serhiyenko, K., Whelan, J., Minimal Length Maximal green Sequences and Triangulations of Polygons, arXiv:1508.02954.

[9] Derksen, H., Weyman, J., and Zelevinsky, A. Quivers with potentials and their representations II: applications to cluster algebras. J. Amer. Math. Soc., 23(3):749-790, 2010.

[10] Fock, V., Goncharov, A., Moduli Spaces of Local Systems and Higher Teichmuller Theory. Publ. Math. Inst. Hautes Etudes Sci. 103 (2006), 1-211.

[11] Fock, V. and Goncharov, A., Dual Teichmüller and lamination spaces. Handbook of Teichmüller theory. Vol. I, 647-684, IRMA Lect. Math. Theor. Phys., 11, Eur. Math. Soc., Zürich, 2007.

[12] Fomin, S., Shapiro, M., and Thurston, D., Cluster Algebras and Triangulated Surfaces Part I: Cluster Complexes, Acta Math. 201 (2008), 83-146.

[13] Fomin, S., and Thurston, D., Cluster algebras and triangulated surfaces. Part II: Lambda Lengths, arXiv: 1210.5569 .

[14] Fomin, S., Zelevinsky, A., Cluster Algebras I: Foundations, Journal of American Mathematical Society. 15 (2002), no. 2, 497-529 (electronic). MR 1887642 (2003f: 16050).

[15] Garver, A., Musiker, G., On Maximal green Sequences for Type A Quivers arXiv:1402.6149.

[16] Gross, M., Hacking, P., Keel, S., Kontsevich, M., Canonical Bases for Cluster Algebras. arXiv:1411.1394v1.

[17] Keller, B., On Cluster Theory and Quantum Dilogarithm Identities, Representation Theory of Algebras and Related Topics (Skowronski, A., Yamagata, K., eds.), European Mathematical Society, (2011), 85-116.

[18] Keller, B., Quiver Mutation and Combinatorial DT-Invariants, corrected version of a contribution to DMTCS Proceedings: FPSAC 2013 (2013).

[19] Kontsevich, M., Soibelman, Y., Stability Structures, Motivic Donaldson-Thomas Invariants and Cluster Transformations, arXiv:0811.2435.

[20] Ladkani, S., On cluster algebras from once punctured closed surfaces, arXiv:1310.4454.

[21] Muller, G., The existence of maximal green sequences is not invariant under mutation, arxiv:1503.04675.

[22] Seven, A., Maximal green sequences of exceptional finite mutation type quivers, SIGMA 10 (2014), 089. 